\title{
Modeling and Design of Single Ring Core DC Sensor based on a NewReadout Method for DC Power System Protection
}

\author{
HU Zhengwei ${ }^{1}$ XIE Zhiyuan ${ }^{1}$ JIN Huiying ${ }^{1}$ PEI Zhaopeng ${ }^{2}$ WANG Lichong ${ }^{2}$ \\ 1 Department of Electronic and Communication,North China Electric Power University, Baoding, China \\ 2 Shenke Electronic Incorporated Company, Hebei Province, China
}

\begin{abstract}
We proposed a new readout method for single ring core direct current (DC) sensor to protect the DC power system. The principle of this readout method is based on the linear relationship between the permeability of magnetic ring core and the DC value when the DC value changes within a small range. We built a Pspice model of single magnetic ring core DC sensor based on the proposed method to prove its correctness. We performed simulations using this model under different conditions, including reference voltage, size of core, turns per coil, excitation period, and sampling frequency. The value of the tested DC can be fitted by one linear equation multiplied by a certain coefficient, and this result was consistent with the principle of the proposed readout method. Several trial manufactures of this sensor participated in a leakage detection test. The sensitivity and noise immunity of physical sensors can be enhanced by using a micro control unit (MCU)embedded system. The correctness of this method can be confirmed using measured data.
\end{abstract}

Key words: DC power system protection, DC sensor, magnetic modulation, leakage current detect

\section{Introduction}

The direct current (DC) power supply system is the basic power source for many devices. Using these devices for a long time will reduce insulation, which may lead to many hidden dangers. Insulation fault may also lead to significant financial losses. To prevent such incidents, insulation-supervising devices have been used to monitor the DC power supply system and send alarm signals to indicate the location of an insulation fault.

The DC sensor is a supervising device that can sample the leakage current and then decide whether to send an alarm or not according to the value of the leakage current.

The DC sensor can be classified as follows: (1) Different voltage level. References [1] to [4] use DC sensors in the ultra-high voltage (UHV) level, while reference[5] uses DC sensors in the low voltage level. (2) Manner of measurement. The DC sensor may be classified into contact and non-contact. The most common non-contact DC sensors are based on the transformation between electric and magnetic. (3) Technologies. The DC sensor may be classified based on electromagnetic induction and other technologies, such as micro-electro-mechanical systems [6]-[8] and opticfiber [9]-[10]. The DC sensor based on electromagnetic induction technology can also be subdivided into hall effect [11]-[12] and magnetic modulation [13]-[16]. (4) Readout strategies. The readout strategy of the DC sensor mainly includes two classifications, namely, second harmonic[17]-[18] and residence times difference (RTD) [19].

We propose a readout method that uses the time difference for measuring DC. This method differs from RTD [19] in several aspects. First, the RTD method involves two coils, namely, the excitation and pickup coils. However, our proposed method uses only one coil to fulfill both the excitation and pickup functions. Figure 1 illustrates such difference. Second, the core works in a saturated state in the RTD method[19] but works in a non-saturated state in the proposed method. Figure 2 illustrates such difference. Third, the proposed method can be combined with a MCU embedded system to enhance sensitivity and noise immunity. 


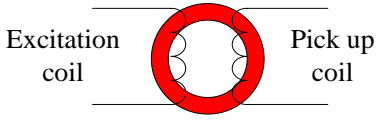

(a) Two coils in the RTD method

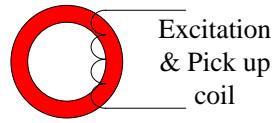

(b) One coil in the proposed method

Figure 1. Differences in coils.

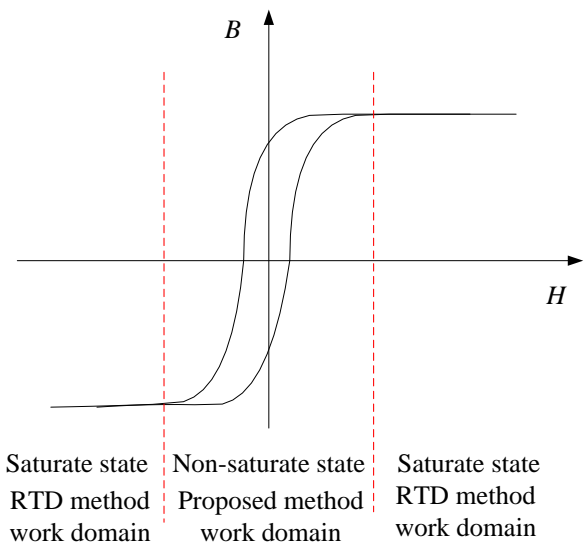

Figure 2. Differences in work domain.

The proposed method can be applied to low voltage level, non-contact, and magnetic modulation DC sensors. Sections 1 and 2 analyze the principle of the proposed readout method for the single ring core DC sensor. Section 3 develops a Pspice model for studying the properties of a DC sensor under different conditions. Section 4 subjects several physical DC sensors to a leakage current detection test to confirm the feasibility of the proposed method. Section 5 concludes the paper.

\section{Analysis of the relationship between inductance and time reaching reference voltage}

Figure 3 presents the schematic of the resistor-inductor $(R L)$ circuit. The clockwise current is in a positive direction.

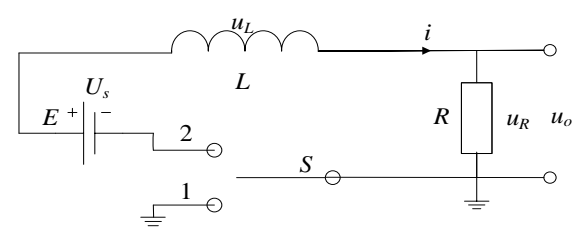

Figure3. Schematic of the RL circuit.

Theoretical analysis:

(1) Initial state $(t<0)$ : The position of the switch is set to 1 .
The inductor is in a steady state, and its resistance can be treated as 0 . The parameters of this state are expressed as follows:

$$
U_{S}=0, u_{L}=0 \text {, and } i=0 \text {. }
$$

(2) Start state $(t=0)$ : The position of the switch is changed from 1 to 2 .

The inductor is in a transient state, and the parameters of this state are expressed as follows:

$$
U_{S}=E, \quad u_{L}=-\varepsilon_{L}=L \frac{d i}{d t}, \text { and } i=\frac{E}{R}\left(1-e^{-\frac{R}{L} t}\right) .
$$

(3) Second state $(t=T / 2)$ : The position of the switch is changed from 2 to 1 .

The inductor is in a transient state, and the parameters of this state are expressed as follows:

$$
U_{S}=0, u_{L}=-\varepsilon_{L}=L \frac{d i}{d t}, \text { and } i=-\frac{E}{R} e^{-\frac{R}{L}\left(t-\frac{T}{2}\right)}
$$

If the first and second states were repeated by cycles $T / 2$ and $T / 2>>L / R$, then the current of circuit $i$ can be described as follows:

$$
i=\left\{\begin{array}{ll}
\frac{E}{R}\left(1-e^{-\frac{R}{L} t}\right) ; & U_{S}=E, t \in\left(0, \frac{T}{2}\right] \\
\frac{E}{R} e^{-\frac{R}{L}\left(t-\frac{T}{2}\right)} ; & U_{S}=0, t \in\left(\frac{T}{2}, T\right]
\end{array} .\right.
$$

The output voltage $u_{o}$ can be computed as $u_{o}=u_{R}=i \times R$, and then $u_{o}$ can be described as follows:

$$
u_{o}=\left\{\begin{array}{ll}
E\left(1-e^{-\frac{R}{L} t}\right) ; & U_{S}=E, t \in\left(0, \frac{T}{2}\right] . \\
E e^{-\frac{R}{L}\left(t-\frac{T}{2}\right)} ; & U_{S}=0, t \in\left(\frac{T}{2}, T\right]
\end{array} .\right.
$$

We can select one voltage value of $u_{o}$ as reference voltage $U_{R E F}$, that is, $U_{R E F} \in u_{o}$. When $U_{R E F}$ is a certain constant value set by the designer, the time $t_{R E F}$ of the output voltage from the starting point to $U_{R E F}$ can be described as follows:

$$
t_{R E F}=\left\{\begin{array}{ll}
\frac{L}{R} \ln \frac{1}{1-\frac{U_{R E F}}{E}} & t_{R E F} \in\left(0, \frac{T}{2}\right] \\
\frac{T}{2}+\frac{L}{R} \ln \frac{E}{U_{R E F}} & t_{R E F} \in\left(\frac{T}{2}, T\right]
\end{array} .\right.
$$

Formula (3) reveals a linear relationship between $t_{R E F}$ and $L$ when the other parameters are determined. We need to measure the value of $t_{R E F}$ and then use formula (3) to obtain the value of the inductor.

Figure 4 shows two waveforms of $u_{\mathrm{o}}$ with 
constant inductor values $L$ and $L^{\prime}$, respectively. $U_{R E F}$ denotes the value of the reference voltage. The starting point denotes the beginning of a new cycle. $t$ and $t^{\prime}$ denote the elapsed time from the starting point to the moment when $u_{\mathrm{o}}$ becomes equivalent to $U_{R E F}$. The difference between $t$ and $t^{\prime}$ can be attributed to the differences of the inductor values.

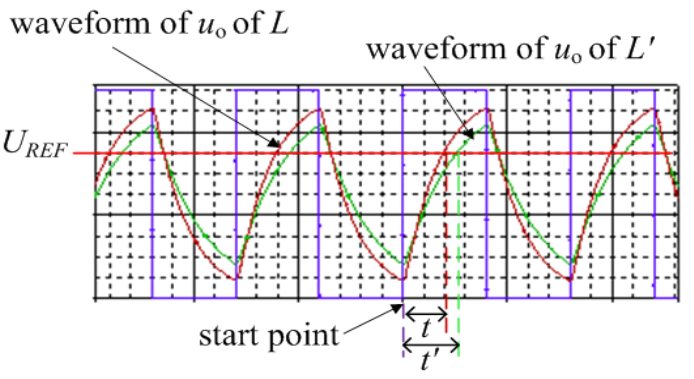

Figure 4. Waveform of $u_{0}$ with different inductor values.

\section{Principle of the proposed readout method}

The DC sensor uses magnetic material as an annular skeleton and is wound with a certain number of turns of wire to form an electromagnetic induction coil. Figure 5 presents the structural diagram of this coil. In this figure, $i$ denotes the excitation alternating current on the wire, and $I$ denotes the DC being measured. The DC sensor based on magnetic modulation can measure $I$ in non-contact mode in which no electric contact point exists between the tested DC and the electromagnetic induction coil.

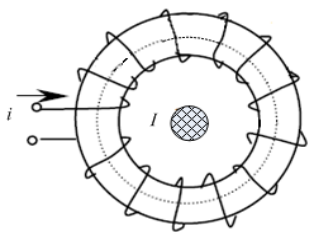

Figure 5. Structural diagram of the electro-magnetic induction coil.

$N$ represents the number of turns of wire, $S$ denotes the cross sectional area, $R_{1}$ and $R_{2}$ denotes the inside and outside radiuses, respectively, and $l_{e}$ denotes the valid length of a magnetic path, which can be described as Formula(4). The inductance value of the coil can be described as Formula(5).

$$
\begin{gathered}
l_{e}=\frac{2 \pi\left(R_{2}-R_{1}\right)}{\ln \frac{R_{2}}{R_{1}}} . \\
L=\frac{N^{2} \cdot S}{l_{e}} \mu .
\end{gathered}
$$

where $\mu$ denotes the magnetic permeability of magnetic core asdefined by Formula(6), $H$ is the magnetic field intensity, and $B$ is the magnetic flux intensity.

$$
\mu=\frac{B}{H} .
$$

According to Ampere's circuital law, the integration of the $H$ inner arbitrary loop is equal to the algebraic sum of all currents inside this loop. This law can be described as follows:

$$
\int_{l} \dot{H} d \dot{l}=\sum_{k} i_{k} .
$$

The magnetic field intensity $H$ of this annulus coil can be described as follows:

$$
\begin{aligned}
H & =\int_{R_{1}}^{R_{2}} H_{r} d r=\int_{R_{1}}^{R_{2}} \frac{N i+I}{2 \pi r} d r=\frac{N i+I}{2 \pi} \int_{R_{1}}^{R_{2}} \frac{1}{r} d r \\
& =\frac{N i+I}{2 \pi}\left(\ln R_{2}-\ln R_{1}\right)=\frac{N i+I}{2 \pi} \ln \frac{R_{2}}{R_{1}}
\end{aligned} .
$$

Figure 6 presents a sketch map of the nonlinear relationship between $\mu$ and $H$. However, if $H$ changes within a small range, such as $H \in\left[0, H_{1}\right]$, then the relationship between $\mu$ and $H$ can be treated as linear.

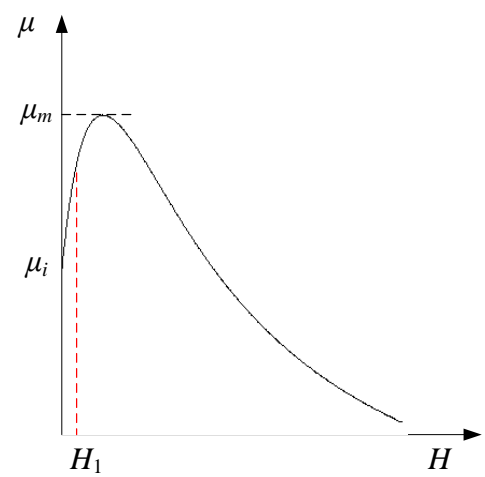

Figure 6. Sketch map of the relationship between $\mu$ and $H$.

To represent this nonlinear relationship, we define the following function:

$$
\mu=g^{\prime}(H) .
$$

We then combine Formulas (9) and (5) as follows:

$$
L=g(H)=\frac{N^{2} \cdot S}{l_{e}} g^{\prime}(H) \text {. }
$$

Formulas (1),(3),(8), and (10)are used to derive an equation set, and only the half cycle $[0, T / 2]$ is listed. 


$$
\left\{\begin{array}{l}
H=\frac{N i+I}{2 \pi} \ln \frac{R_{2}}{R_{1}} \\
L=g(H)=\frac{N^{2} \cdot S}{l_{e}} g^{\prime}(H) \\
t_{R E F}=\frac{L}{R} \ln \frac{1}{1-\frac{U_{R E F}}{E}} \\
i=\frac{E}{R}\left(1-e^{-\frac{R}{L} t}\right)
\end{array}\right.
$$

The above equation set reveals that the variation of $I$ results in the variation of $H[$ Formula (8)], that the variation of $H$ results in the variation of $L$ [Formula (10)], and that the variation of $L$ results in the variation of $t_{R E F}$. Therefore, the variation of $I$ results in the variation of $t_{R E F}$. The relationship between $I$ and $t_{R E F}$ can be described by Formula(11). At the moment of $t_{R E F}, \quad i=\frac{U_{R E F}}{R}$.

$$
\begin{aligned}
& t_{R E F}=\frac{L}{R} \ln \frac{1}{1-\frac{U_{R E F}}{E}} \\
& =\frac{N^{2} \cdot S}{R \cdot l_{e}} g^{\prime}(H) \cdot \ln \frac{1}{1-\frac{U_{R E F}}{E}} \\
& =\frac{N^{2} \cdot S}{R \cdot l_{e}} \cdot \ln \frac{1}{1-\frac{U_{R E F}}{E}} \cdot g^{\prime}\left(\frac{N \frac{U_{R E F}}{R}+I}{2 \pi} \ln \frac{R_{2}}{R_{1}}\right) . \\
& =k_{t} \cdot h(I)
\end{aligned} .
$$

From the above formula, we propose the following definitions:

$$
\begin{gathered}
k_{t}=\frac{N^{2} \cdot S}{R \cdot l_{e}} \cdot \ln \frac{1}{1-\frac{U_{R E F}}{E}} \text { and } \\
g^{\prime}\left(\frac{N \frac{U_{R E F}}{R}+I}{2 \pi} \ln \frac{R_{2}}{R_{1}}\right)=h(I) .
\end{gathered}
$$

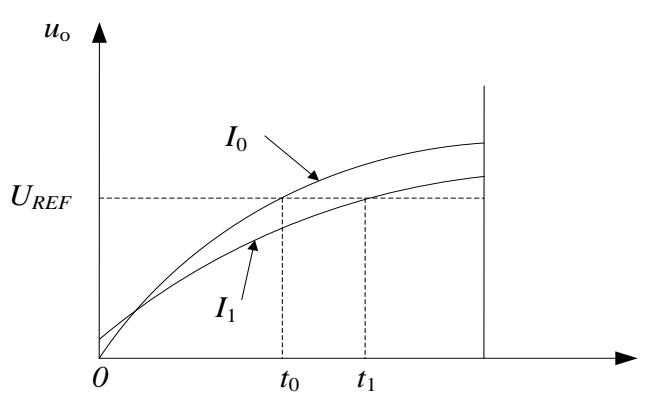

Figure 7. Time measurement principle.

Figure 7 presents two curves of $u_{o}$, with each curve being associated with a different $\mathrm{DC}$ value. $t_{R E F}=t_{1}$ when $I=I_{1}$, and $t_{R E F}=t_{0}$ when $I=I_{0}=0 . t_{0}$ and $t_{1}$ can be measured, and the difference between them can be calculated as follows:

$$
t_{1}-t_{0}=k_{t}\left[h\left(I_{1}\right)-h(0)\right] .
$$

$I_{1}$ can be calculated as follows:

$$
I_{1}=h^{-1}\left(\frac{t_{1}-t_{0}}{k_{t}}+h(0)\right) .
$$

where $h^{-1}(\cdot)$ is the anti-function of $h(\cdot)$.

The function $t_{R E F}=k_{t} \cdot h(I)$ must be determined before calculating the value of $I$.

Figure 6 reveals that when $H \in\left[0, H_{1}\right]$, the relationship between $\mu$ and $H$ can be treated as linear. If the DC sensor works in $H \in\left[\begin{array}{ll}0, & H_{1}\end{array}\right]$ and $\mu=g^{\prime}(H)=k H$ where $k$ is a constant, then

$$
h(I)=k \frac{N \frac{U_{R E F}}{R}+I}{2 \pi} \ln \frac{R_{2}}{R_{1}} .
$$

According to Formula (11), $t_{R E F}=k_{t} h(I)$.

$$
\begin{aligned}
& t_{R E F}=k_{t} h(I)=k_{t} k \frac{N \frac{U_{R E F}}{R}+I}{2 \pi} \ln \frac{R_{2}}{R_{1}} \\
& t_{1}=k_{t} h\left(I_{1}\right)=k_{t} k \frac{N \frac{U_{R E F}}{R}+I_{1}}{2 \pi} \ln \frac{R_{2}}{R_{1}} \\
& t_{0}=k_{t} h(0)=k_{t} k \frac{N \frac{U_{R E F}}{R}}{2 \pi} \ln \frac{R_{2}}{R_{1}}
\end{aligned}
$$




$$
\begin{aligned}
& t_{1}-t_{0}=I_{1} \cdot \frac{k_{t} k}{2 \pi} \ln \frac{R_{2}}{R_{1}} \\
& =-I_{1} \cdot k \frac{N^{2} \cdot S}{2 \pi R l_{e}} \ln \left(\frac{R_{2}}{R_{1}}\right) \ln \left(1-\frac{U_{R E F}}{E}\right) \\
& I_{1}=-\frac{t_{1}-t_{0}}{k \frac{N^{2} \cdot S}{2 \pi R l_{e}} \ln \left(\frac{R_{2}}{R_{1}}\right) \ln \left(1-\frac{U_{R E F}}{E}\right)} .
\end{aligned}
$$

The above formula shows that the relationship between the DC value and the time difference can use a linear function to demonstrate if the relationship between $\mu$ and $H$ is linear.

\section{Modeling of single-core DC sensor based on the proposed read out method}

We built a model of the single ring core DC sensor based on proposed readout method by using the Pspice software. We initially investigate the relationship between $\mu$ and $H$ before performing simulations under different conditions, including reference voltage, material, size of coil, turns per coil, excitation cycle, and sampling frequency.

\subsection{Modeling of single core DC sensor}

The measurement circuit of a single ring core DC sensor can be built based on the basic $R L$ circuit by adding the magnetic ring core and the modulation circuit. Figure 8 shows the schematic of the single ring core DC sensor.

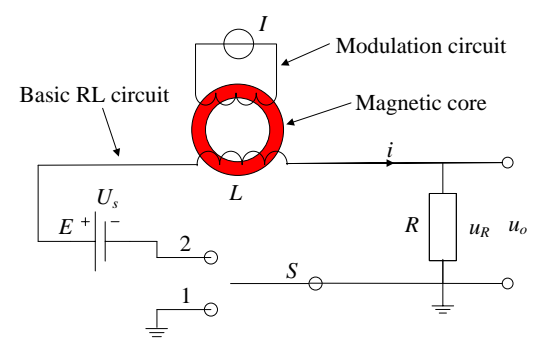

Figure 8. Schematic of the single core DC sensor.

On the basis of the above schematic,we build a Pspice model of the single core DC, as shown in Figure 9. This model can perform many simulations under different conditions without using physical hardware.

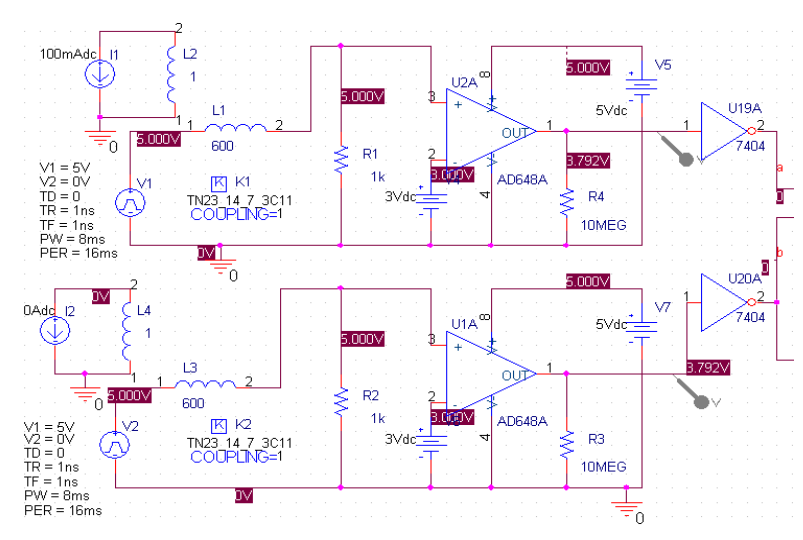

(a) Part one

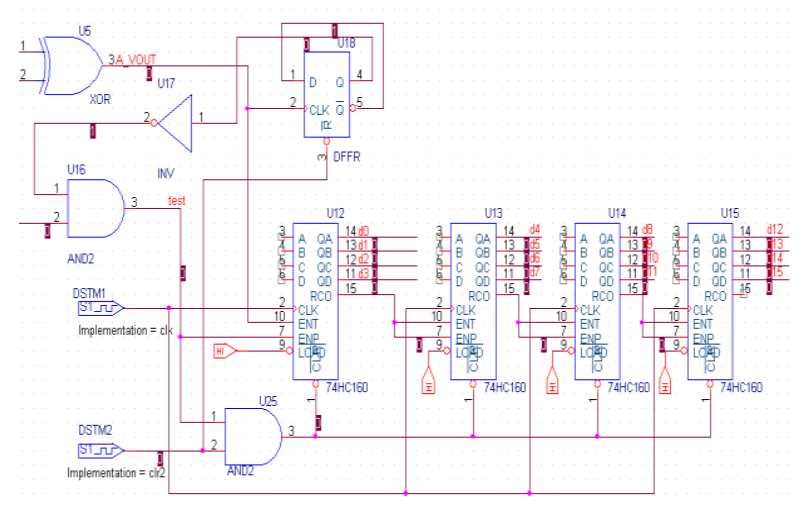

(b) Part two

Figure 9. Pspice model of the single core DC sensor.

Figure 9 divides the model into two sub-figures that can be connected as follows: (1) The 2 pin of U19A in part one must be connected with the 1 pin of U5 in part two; (2) the 2 pin of U20A in part one must be connected with the 2 pin of U5 in part two; and (3) the 2 pin of U20A in part one must be connected with the 2 pin of U16 in part two.

We use the DC sweep function of the Pspice software to investigate the relationship between the $\mu$ and $H$ of three types of ring cores, namely, TN32_19_13_3C11, TN23_14_7_3C11, and TN23_14_7_3C85. Table 1 lists the parameters of these ring cores.

Table 1. Parameters of three magnetic cores

\begin{tabular}{|c|c|c|c|}
\hline & TN23_14_7_3C11 & TN23_14_7_3C85 & TN32_19_13_3C11 \\
\hline MS & $324.29 \times 10^{3} \mathrm{~A} / \mathrm{m}$ & $377.33 \times 10^{3} \mathrm{~A} / \mathrm{m}$ & $324.29 \times 10^{3} \mathrm{~A} / \mathrm{m}$ \\
\hline $\mathrm{A}$ & $16.458 \mathrm{~A} / \mathrm{m}$ & $22.210 \mathrm{~A} / \mathrm{m}$ & $16.458 \mathrm{~A} / \mathrm{m}$ \\
\hline $\mathrm{C}$ & 0.27883 & 0.13336 & 0.27883 \\
\hline $\mathrm{K}$ & $11.341 \mathrm{~A} / \mathrm{m}$ & $20.004 \mathrm{~A} / \mathrm{m}$ & $11.341 \mathrm{~A} / \mathrm{m}$ \\
\hline AREA & $0.309 \mathrm{~cm}^{2}$ & $0.309 \mathrm{~cm}^{2}$ & $0.765 \mathrm{~cm}^{2}$ \\
\hline PATH & $5.5800 \mathrm{~cm}$ & $5.5800 \mathrm{~cm}$ & $7.6 \mathrm{~cm}$ \\
\hline
\end{tabular}

Figures10(a) and 10(b) show that the sweep 
ranges of the current are $[-10 \mathrm{~A},+10 \mathrm{~A}]$ and [-100 mA,+100mA] respectively. Figure10(a) shows a nonlinear relationship withina large current range, while Figure 10(b) shows a linear relationship within a small current range.

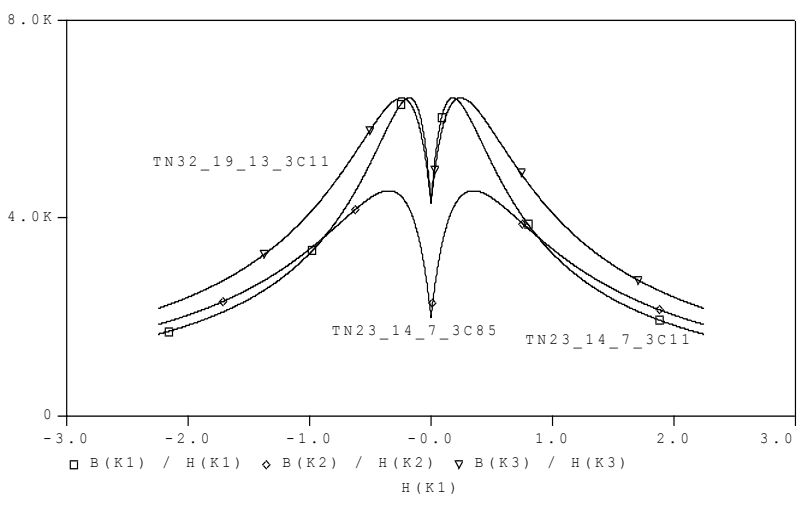

(a) Current range in $[-10 \mathrm{~A},+10 \mathrm{~A}]$

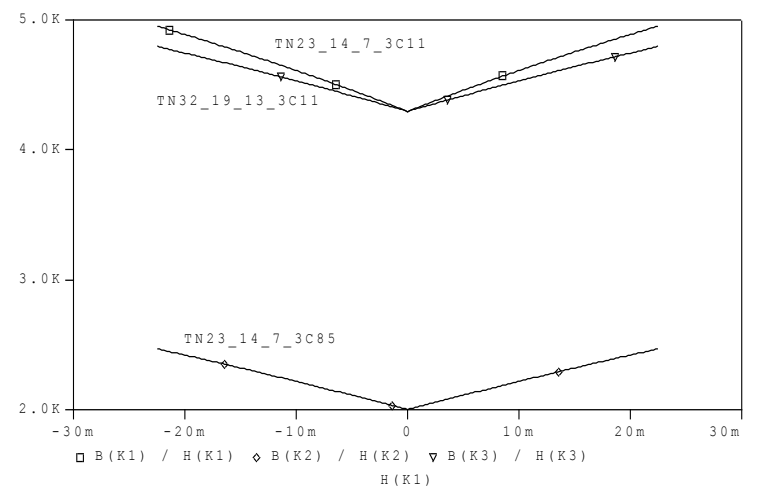

(b) Current range in $[-100 \mathrm{~mA},+100 \mathrm{~mA}]$

Figure 10. Relationship between $\mu$ and $H$.

\subsection{Analysis of the simulation results}

Table 2 lists the simulation conditions. We use aliases for each condition, such as"type of magnetic core_size of external diameter_reference voltage_ turns_excitation period_sample frequency." For example, we use "3C11_23_2V_300_16ms_8M" to indicate condition(1) as listed in Table2.
Table 2. Simulation conditions

\begin{tabular}{|c|c|c|c|c|c|c|}
\hline & $\begin{array}{c}\text { Magnetic } \\
\text { core }\end{array}$ & Size & $\begin{array}{c}\text { Reference } \\
\text { voltage }\end{array}$ & Turns & $\begin{array}{c}\text { Excitation } \\
\text { period }\end{array}$ & $\begin{array}{c}\text { Sample } \\
\text { frequency }\end{array}$ \\
\hline (1) & $3 \mathrm{C} 11$ & $23 \_14 \_7$ & $2 \mathrm{~V}$ & 300 & $16 \mathrm{~ms}$ & $8 \mathrm{MHz}$ \\
\hline (2) & $3 \mathrm{C} 11$ & $23 \_14 \_7$ & $3 \mathrm{~V}$ & 300 & $16 \mathrm{~ms}$ & $8 \mathrm{MHz}$ \\
\hline (3) & $3 \mathrm{C} 11$ & $23 \_14 \_7$ & $2 \mathrm{~V}$ & 600 & $16 \mathrm{~ms}$ & $8 \mathrm{MHz}$ \\
\hline (4) & $3 \mathrm{C} 11$ & $23 \_14 \_7$ & $3 \mathrm{~V}$ & 600 & $16 \mathrm{~ms}$ & $8 \mathrm{MHz}$ \\
\hline (5) & $3 \mathrm{C} 11$ & $23 \_14 \_7$ & $3 \mathrm{~V}$ & 600 & $16 \mathrm{~ms}$ & $16 \mathrm{MHz}$ \\
\hline (6) & $3 \mathrm{C} 85$ & $23 \_14 \_7$ & $3 \mathrm{~V}$ & 600 & $16 \mathrm{~ms}$ & $16 \mathrm{MHz}$ \\
\hline (7) & 3C11 & $23 \_14 \_7$ & $3 \mathrm{~V}$ & 600 & $8 \mathrm{~ms}$ & $16 \mathrm{MHz}$ \\
\hline (8) & 3C11 & $32 \_19 \_13$ & $3 \mathrm{~V}$ & 600 & $16 \mathrm{~ms}$ & $16 \mathrm{MHz}$ \\
\hline
\end{tabular}

Figure 10(b) shows that the DC for the simulation ranges from $0 \mathrm{~mA}$ to $100 \mathrm{~mA}$ with a $5 \mathrm{~mA}$ step. We use a linear equation to fit the simulation results, that is, we suppose that the relationship between $t_{R E F}$ and $I$ can be described using a linear equation.

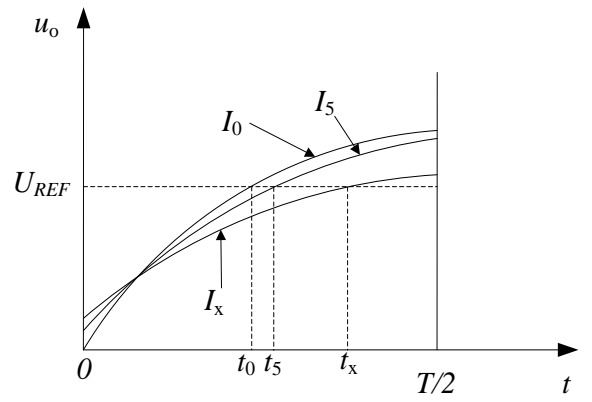

Figure 11. Principle of the linear fitted equation.

Figure 11 shows the three curves of $u_{0} . I_{x}$ indicates $I=x$, and $t_{x}$ indicates the value of $t_{R E F}$ when $I=x . I_{x}$ can be calculated as follows:

$$
I_{x}=I_{x}-I_{0}=\frac{5}{t_{5}-t_{0}}\left(t_{x}-t_{0}\right),
$$

where $t_{5}$ and $t_{0}$ are treated as known quantities, $t_{x}$ denotes the measured value, and $I_{x}$ denotes the calculated value. Fitting error $I_{e}$ can be calculated as follows:

$$
I_{e}=I_{x}-x .
$$

The measured value $t_{x}$ based on the Pspice model represents the average value of $10 t_{x}$ samples.

Figure 12 shows the fitting errors of Formula (16). These errors increase along with $I$ and reach their peak at approximately $10 \mathrm{~mA}$, as shown in Figure 12. 


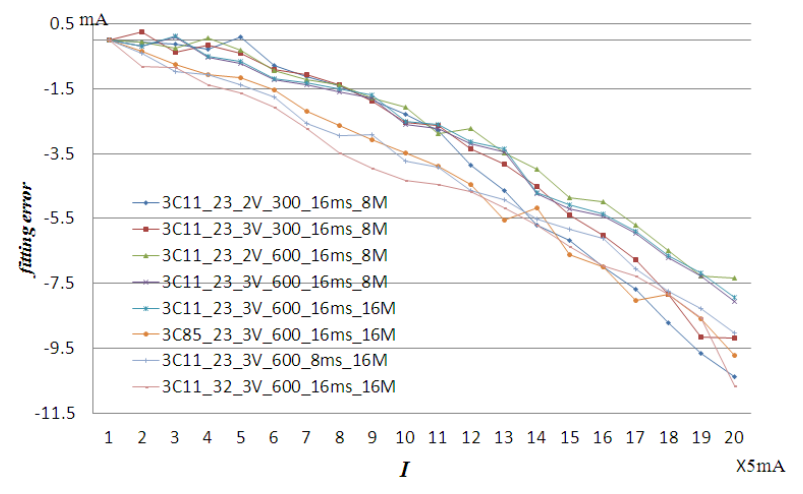

Figure 12. Fitting errors of Formula (16).

$$
I_{x}=I_{x}-I_{0}=\frac{5}{0.92\left(t_{5}-t_{0}\right)}\left(t_{x}-t_{0}\right)(17)
$$

Figure 13 shows the fitting errors when the above formula is used as the fitting equation. The fitting errors in Figure 13 are improved compared with those in Figure12. The largest fitting error in Figure 13 is approximately $2.6 \mathrm{~mA}$.

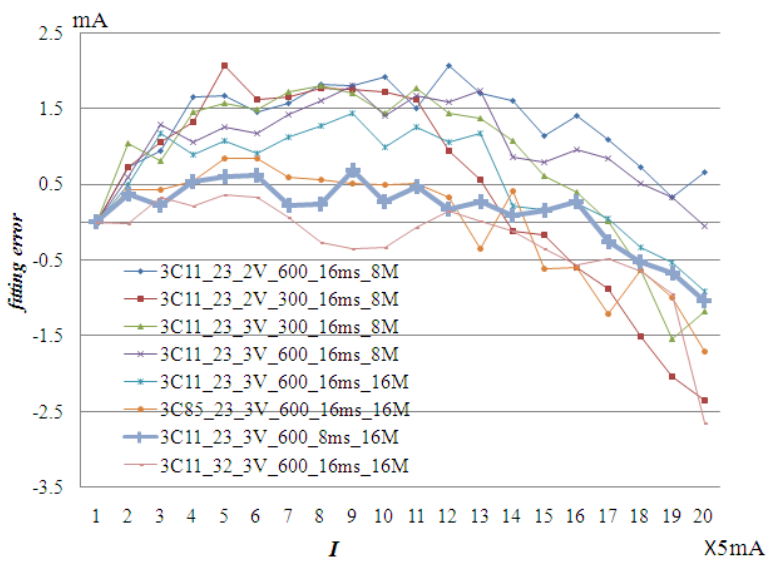

Figure 13. Fitting errors of Formula(17).

Sensitivity is another important factor that must be considered. This factor pertains to the minimum measurable current.

$$
\begin{aligned}
& t_{x 1}-t_{0}=-k \frac{N^{2} \cdot S}{2 \pi R l_{e}} \ln \left(\frac{R_{2}}{R_{1}}\right) \ln \left(1-\frac{U_{R E F}}{E}\right) \cdot I_{x 1} \\
& t_{x 2}-t_{0}=-k \frac{N^{2} \cdot S}{2 \pi R l_{e}} \ln \left(\frac{R_{2}}{R_{1}}\right) \ln \left(1-\frac{U_{R E F}}{E}\right) \cdot I_{x 2} \\
& t_{x 2}-t_{x 1}=-k \frac{N^{2} \cdot S}{2 \pi R l} \ln \left(\frac{R_{2}}{R_{1}}\right) \ln \left(1-\frac{U_{R E F}}{E}\right) \cdot\left(I_{x 2}-I_{x 1}\right)
\end{aligned}
$$

The sensitivity is controlled by the coefficient of the following equation:

$$
k_{s}=-k \frac{N^{2} \cdot S}{2 \pi R l_{e}} \ln \left(\frac{R_{2}}{R_{1}}\right) \ln \left(1-\frac{U_{R E F}}{E}\right) .
$$

Figure 14 illustrates the sensitivity of all conditions. Condition

“3C11_32_3V_600_16ms_16M ” achieves the maximum sensitivity.

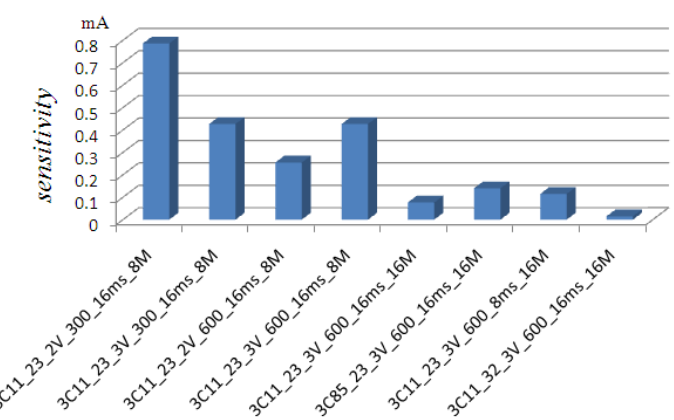

Figure 14. Sensitivity of different conditions.

\section{Implementation and test of the single core DC} sensor based on the proposed readout method

4.1 Implementation of the physical DC sensor

We trial-manufacture several physical single ring core DC sensors based on the proposed readout method to conduct a leakage protection test. Figure 15 shows the components of a single ring core DC sensor.

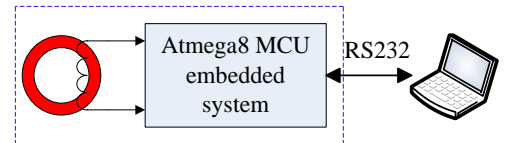

Figure 15. Components of single-core DC sensor.

The Atmega8 MCU embedded system offers two advantages. First, the sensitivity of the sensor can be enhanced by multiplying a coefficient greater than 1 by $k_{s}$ as defined in Formula(19). Second, the stability of the readout data can be enhanced by averaging many sampled data.

(1) Enhancement of sensitivity

We deduce the following equation according to Formulas(18) and (19):

$$
\Delta t=k_{s} \cdot \Delta I .
$$

For a DC sensor, the value of $k_{s}$ is constant, and the sensitivity is fixed.

Formula (20) can be transformed as follows:

$$
\Delta t=k_{s} \cdot C \cdot \frac{\Delta I}{C}=C \cdot k_{s} \cdot \Delta I^{\prime},
$$

where $\Delta I^{\prime}=\frac{\Delta I}{C}$.

If $C>1$, then $\Delta I^{\prime}<\Delta I$. Therefore, a smaller DC value can be detected.

On the basis of this conclusion, a coefficient $C$ 
greater than 1 can be multiplied by $k_{s}$ to enhance the sensitivity.

(2) Enhancement of the stability of the readout data

The average operation can smoothen the sample data based on the followingprinciple:

$$
\bar{t}=\frac{1}{N_{T}} \sum_{i=1}^{N_{T}} t(i),
$$

where $T_{s}$ is the period of excitation, $N_{T}$ is the number of periods of excitation, $t(i) \quad\left(\mathrm{i} \in\left[1, N_{T}\right]\right)$ represents the readout data of one $T_{s}, \bar{t}$ is the readout data of one period equal to $N_{T} \cdot T_{s}$, and $\bar{t}$ is the average value of $t(i)$ that fluctuatesless than each $t(i)$.

Apart from smoothening the sample data, the average operation can also improve noise immunity.

The main noises in the DC power system come from the $\mathrm{AC}$ power system in the form of 50 or 60 Hz sinusoid signals.

If $T_{n}$ is the period of interference noise, then $N_{T n}$ is the number of $T_{n}$ in the period of $N_{T} \cdot T_{s} . N_{T n}$ can be calculated as follows:

$$
N_{T n}=\frac{N_{T} \cdot T_{s}}{T_{n}} .
$$

For the periodic sinusoid signal, the average value of one period is 0 . Therefore, the average value of $N_{T n}$ periods is also 0 .

$T_{s}$ and $N_{T}$ must be selected properly to guarantee noise immunity.

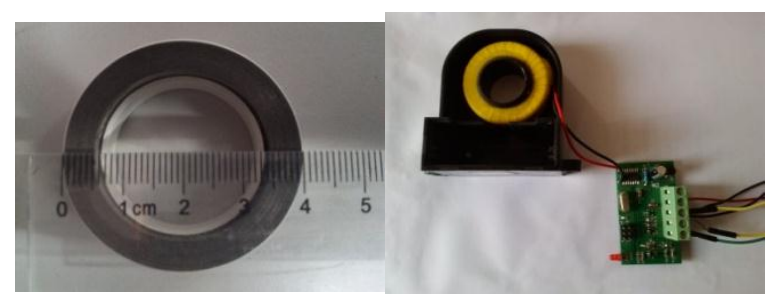

(a) Magnetic core

(b) WholeDC sensor

Figure 16. Trial-manufactured DC sensor.

Figure 16 shows a physical sensor; its coil is produced by Hebei Shenke Electronics Co. Ltd. Table 3 lists the characteristics of the physical sensor.
Table 3 Characteristicsof the physical sensor

\begin{tabular}{|c|c|c|c|c|c|}
\hline $\begin{array}{c}\text { Magnetic } \\
\text { core }\end{array}$ & Size & $\begin{array}{c}\text { Reference } \\
\text { voltage }\end{array}$ & Turns & $\begin{array}{c}\text { Excitation } \\
\text { period }\end{array}$ & $\begin{array}{c}\text { Samplefre } \\
\text { quency }\end{array}$ \\
\hline FeSiB & $40 \_30 \_10$ & $3 \mathrm{~V}$ & 300 & $16 \mathrm{~ms}$ & $4 \mathrm{MHz}$ \\
\hline
\end{tabular}

Figure 17 shows the hysteresis loop of a ferromagnetic core.This core will work in a saturated state if the magnetic field intensity $H$ is greaterthan $15 \mathrm{~A} / \mathrm{m}$ or less than $-15 \mathrm{~A} / \mathrm{m}$.

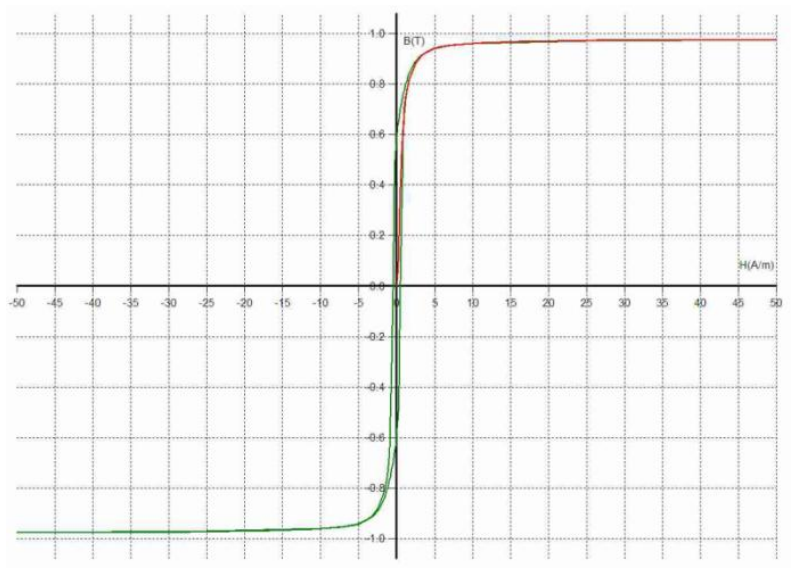

Figure 17. Hysteresis loop of a ferromagnetic core.

The test range of the leakage current is less than $80 \mathrm{~mA}$, the maximum value of the excitation current is $5 \mathrm{~mA}$, the maximum value of the total current is less than $85 \mathrm{~mA}$, the valid length of the magnetic path is approximately $106.5 \mathrm{~mm}$, and the magnetic field intensity is less than $0.8 \mathrm{~A} / \mathrm{m}$. According to the hysteresis loop of the ferromagnetic core, the DC sensor does not work in a saturated state. The excitation current has a range of $[-5 \mathrm{~mA}, 5 \mathrm{~mA}]$, whereas the magnetic field intensity produced by the excitation current has an excitation swing of [-0.045 A/m, $0.045 \mathrm{~A} / \mathrm{m}]$.

\subsection{Test and result analysis}

We perform the test after using all sensors for approximately 96 hours. The tested DCs have values of $0,2,5,10,20,40$, and $80 \mathrm{~mA}$. For each sensor, the readout data represent the average value of 100 sampled data, that is, $N_{T}=100$. The fitting equation is similar to Formula(15). We use the fitting errors to confirm the validity of the proposed method. The results of each test are the average values of eight sensors. To enhance sensitivity, we multiply a constant equal to 10000 by $k_{s}$. 


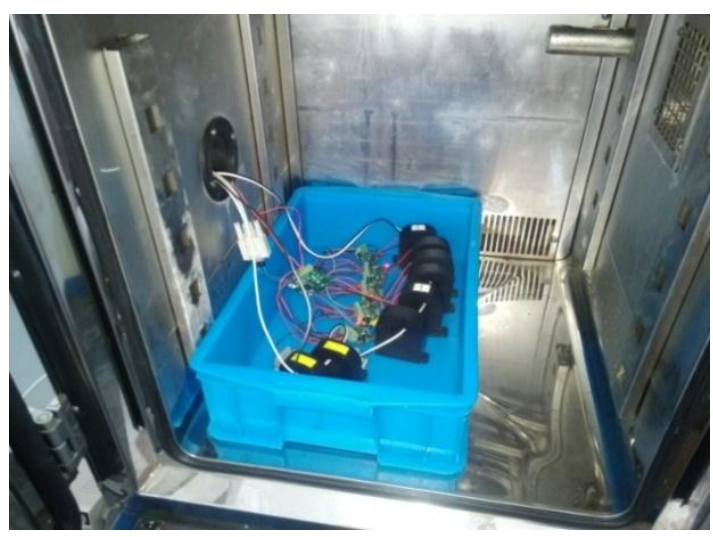

(a) Innerthermostat of eight sensors

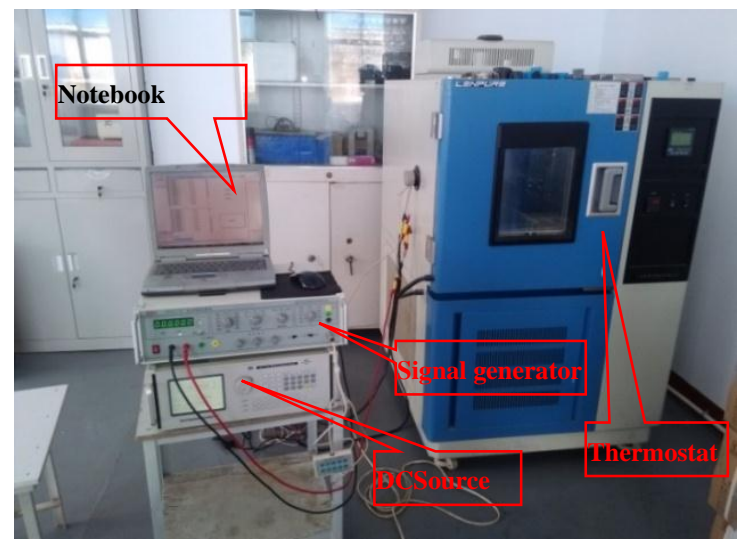

(b) Test system

Figure 18. Test system of the single-core DC sensor based on the proposed readout method.

As shown in Figure18(a), we place eight sensors in the thermostat and then set the working temperature. Figure 18(b) shows the whole test system. The test system includes the DC source, signal generator, and notebook aside from the thermostat. We use the signal generator to generate an interference signal.

(1) Linear and temperature characteristics

According to Formula(18), the linearity was determined by $k$, which was controlled by the magnetic ring core. The temperature dependence of this DC sensor was also controlled by the material of the magnetic core and could be resolved by selecting the magnetic core with the minimum temperature dependence.

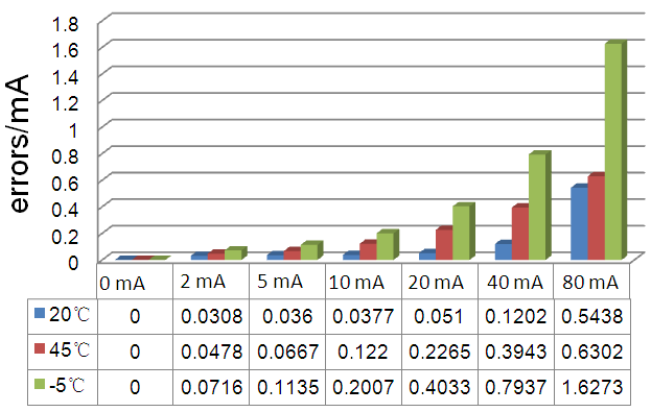

Figure 19. Linear and temperature characteristics.

Figure 19 shows the fitting errors under temperatures of 20,45 , and $-5{ }^{\circ} \mathrm{C}$. Given that we use Formula (15) as a linear fitting equation, the linear characteristics under each temperature can be confirmed in the test DC range.

(2) Noise immunity characteristics

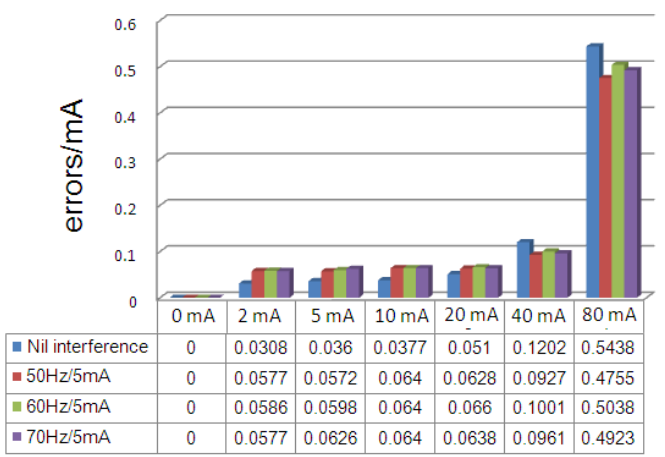

(a) $5 \mathrm{~mA}$

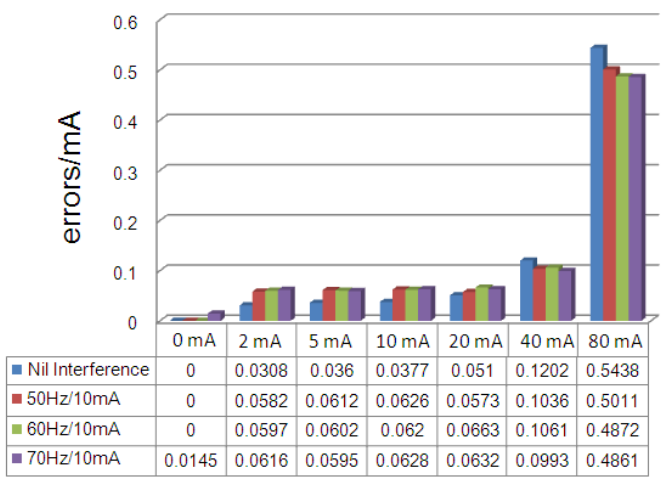

(b) $10 \mathrm{~mA}$

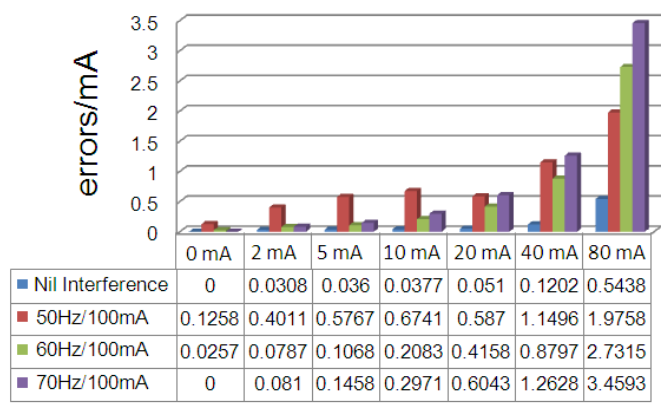

(c) $100 \mathrm{~mA}$

Figure 20 . Immunity characteristics in $20^{\circ} \mathrm{C}$. 


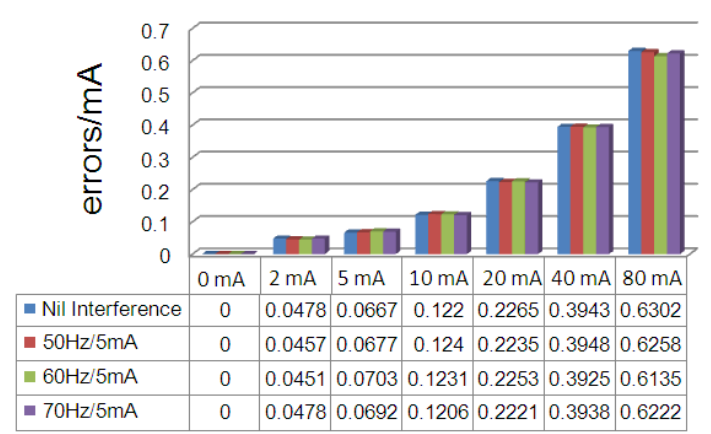

(a) $5 \mathrm{~mA}$

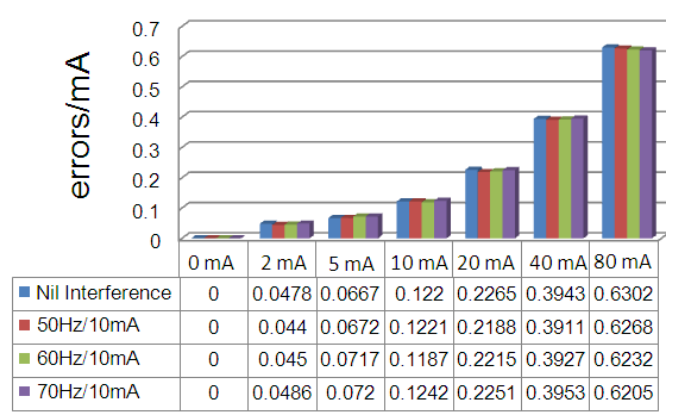

(b) $10 \mathrm{~mA}$

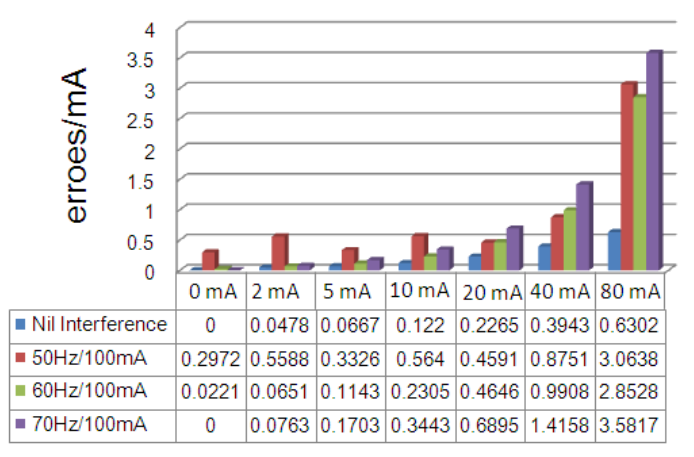

(c) $100 \mathrm{~mA}$

Figure 21. Immunity characteristics in $45^{\circ} \mathrm{C}$

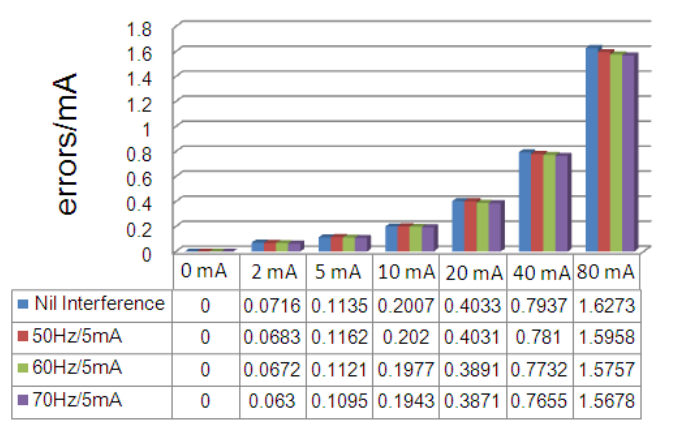

(a) $5 \mathrm{~mA}$

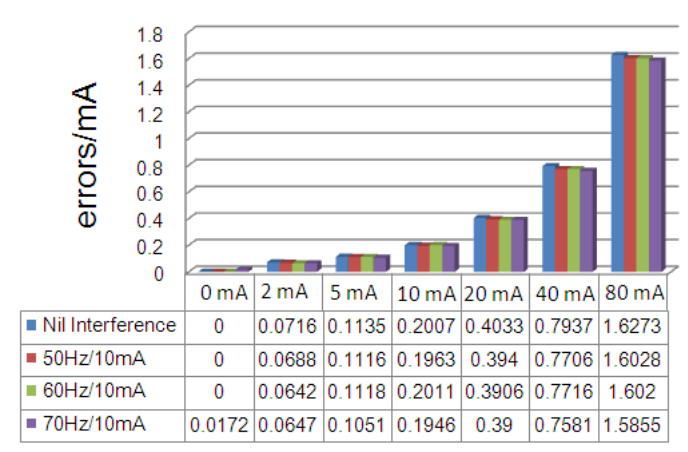

(b) $10 \mathrm{~mA}$

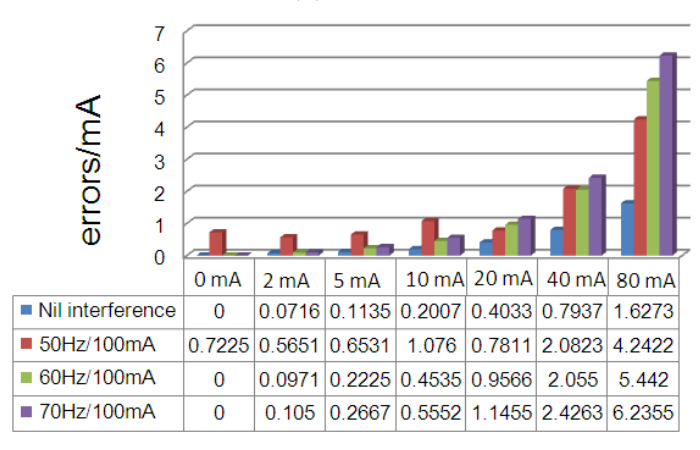

(c) $100 \mathrm{~mA}$

Figure 22. Immunity characteristics in $-5^{\circ} \mathrm{C}$.

Figures 20, 21, and 22 show the noise immunity characteristics of the sensors under three different temperatures.

According to Formula(23), if $N_{T}=100, T_{s}=16 \mathrm{~ms}$, the valule of $N_{T n}$ is 80,96 , and 112 when surfering from interference of 50,60, and $70 \mathrm{~Hz}$ respectively. Therefore, the average operation of the MCU embedded system enhances the noise immunity characteristic.

However, the worst immunity characteristics are achieved at $50 \mathrm{~Hz} / 100 \mathrm{~mA}$ under a small DC value. The reasons behind this strange phenomenon warrant further study.

Figures 19, 20, 21, and 22 show that the proposed DC sensors can detect the target DC value with small fitting errors and can be used for detecting the leakage current of the DC power system.

\section{5 . Conclusion}

We propose a single ring core DC sensor based on a new readout method to detect the leakage current of the DC power system. The proposed method argues that the relationship between $\mu$ and $H$ is linear within a small current range. This principle suggests that the relationship between time reach to reference voltage and target $\mathrm{DC}$ value is also linear. 
We build a Pspice model based on the proposed method and then perform simulations under several conditions. We multiply a linear equation by a coefficient to create a fitting equation and to control the fitting errors within a small range.

We confirm the feasibility and effectiveness of our proposed method by implementing several physical sensors based on this method.

However, many other problems warrant further study, such as how the influence of temperature and big noise can be deduced, as well as the characteristics of the magnetic core with minimal temperature dependence.

\section{Acknowledgement}

This work was supported by the National Science Foundation of China (No.61172075) and the Fundamental Research Funds for the Central Universities (No.2015MS98).

\section{References}

[1] Ye FEI, Xiao-qi WANG, Shi-pu WU, etc.Skin Effect Analysis and Structure Optimization of $\pm 1000 \mathrm{kV}$ UHVDC Current Transformer[J].High Voltage Engineering,

2011,37(2),361-367( in chinese)

[2]Ye FEI, Xiao-qi WANG, Ben-jin WANG, etc. Development on $\pm 1000 \mathrm{kV}$ UHVDC instrument transformer [J].High Voltage Engineering, 2010,36(10):2380-2387( in chinese)

[3]Wen YANG, Jiang-li BAO, Yan-jie HOU, etc.Development of $\pm 800 \mathrm{kV}$ DC Electronic Current Transformer[J]. High Voltage Apparatus, 2011,47(1):10-17 (in chinese)

[4]Qian LI, He LI, Yi-fei ZHOU,etc.On-site Calibration Technology of DC Current Measurement Device in Converter Station of $\pm 800 \mathrm{kV}$ UHVDC Transmission Project[J]. .High Voltage Engineering, 2011,37(12):3053-3058(in chinese)

[5]Feng-kai YANG.Development of Magnetic Modulation Active Sensor for Small DC Current

Measurement[J].InstrumentTechniqueandSensor,

2008,10,9-11(in chinese)

[6]Kohei Isagawa, DongF. Wang, Takeshi Kobayashi, etc. Development of A MEMS DC Electric Current Sensor Applicable to Two-wire Electrical Appliance Cord[J].Nano/Micro Engineered and Molecular Systems (NEMS), 2011 IEEE International Conference on, 932-935.

[7]Kohei Isagawa, Dong F. Wang, Takeshi Kobayashi, etc. Developing MEMS DC Electric Current Sensor for End-use
Monitoring of DC Power Supply: Part II MEMS-scale Device with Five-PZT Plates[J].Design, Test, Integration and Packaging of MEMS/MOEMS (DTIP), 2012 Symposium on, 244-247.

[8]Yasuhiro Suzuki, Dong F. Wang, Takeshi Kobayashi, etc. Developing MEMS DC Electric Current Sensor for End-use Monitoring of DC Power Supply: Part III - Integration with Actuating and Sensing Elements[J].Design, Test, Integration and Packaging of MEMS/MOEMS (DTIP), 2013 Symposium on, 1-4.

[9]Klaus Bohnert, Philippe Gabus, Hubert Brändle and etc. Fiber-optic dc current sensor for the electro-winning industry[J]. 17th Int. Conference on Optical Fibre Sensors, Bruges, Belgium, May 23-27, 2005:210-213

[10] Marc Wendler. New DC current measurement technology with fiber-optic sensor[ob/dl]. Energize,Jan/Feb, 2005:46-47

[11]Vincent Mosser, Youcef Haddab. Real-time broadband subnoise level signal extraction in Hall sensors[J]. Noise and Fluctuations (ICNF), 2013 22nd International Conference on, $1-4$.

[12]James Lenz, Alan S Edelstein. Magnetic sensors and their applications[J].IEEE Sensors Journal,2006, 6(3):631 649.

[13] A.Q. Ma, S.Y. Ren, X.C. Jiang and etc. DC sensor based on magnetic potential self-balance and feedback compensation[J].IET Science Measurement \&Technology, 2009, 3(4):312-316, .

[14]Cheng-Fa Cheng, Rong-Sing Li, yue-Ru Chen. Design of the DC Leakage Current Sensor with Magnetic Modulation-Based Scheme[J].Industrial Electronics (ISIE), 2013 IEEE International Symposium on, 1-6.

[15]Ca-i Hong ZHAO, Wen-Li SHENG, Zh-i Yong XU. The Develop Of InSulation Monitor On DC System By Magnetic Modulation[J]. Journal Of Northeast China Institute Of Electric Power Engineering. 2001,21(4):13-16,33.(in chinese) [16]Min ZHANG, Xiang-jun WANG, Dou JI, etc. Mathematical model and performance analysis of magnetic modulated current sensor[J].JOURNAL OF NAVAL U NIVERSITY OF ENGINEERING.2008,20(6):75-78(in chinese).

[17] F. Forster, A method for the measurement of dc field differences and its application to nondestructive testing,[J].

Nondestruct Test, 1955,13:31-41

[18] F. Primdahl. The fluxgate mechanism, part I: the gating curves of parallel and orthogonal fluxgates[J]. IEEE Trans. Magn, 1970,6(2):376-383 
[19]B. Andò, S. Baglio, A. Bulsara, V. Sacco, Residence

Times Difference fluxgate magnetometers[J]. Sens. J. IEEE

2005,5 (5):895-904 
Zhengwei HU received the M.S. in telecomunication engineering from North china electric power university in 2004 and received Ph.D. degrees in microelectronics from beijing institute of technology in 2007. His research activity is focused on DC sensor design for protection of power system. 